\title{
A three-and-a-half-year-old patient with Prader-Willi syndrome: a case report on dental management with behavioral modification
}

\author{
Vignesh Guptha Raju ${ }^{*}$, Muralidharan Dhanasekaran ${ }^{2}$, Jayanthi Mungara ${ }^{3}$, Deepak Velu ${ }^{4}$, Vivek Rajashekar ${ }^{5}$ and Harish Sureshbabu $^{6}$ \\ ${ }^{1}$ Department of Pedodontics and Preventive Dentistry, Karpaga vinayaga institute of dental sciences, Tamil Nadu, India \\ ${ }^{2}$ Department of Orthodontics, Karpaga vinayaga institute of dental sciences, India \\ ${ }^{3}$ Department of Pedodontics and Preventive Dentistry, Ragas dental college and hospital, India \\ ${ }^{4}$ Department of Oral and maxillofacial surgery, Karpaga vinayaga institute of dental sciences, India \\ ${ }^{5}$ Department of Oral medicine and Radiology, Karpaga vinayaga institute of dental sciences, India \\ ${ }^{6}$ Internship at Karpaga vinayaga institute of dental sciences, India
}

\begin{abstract}
Background: Early diagnosis and intervention play an important role in prognosis while treating an infant and an young child particularly, associated with some syndrome.

Purpose: This article deals with a case report of a three-and-a-half-year-old male child with Prader-Willi syndrome (PWS) in whom the risk of caries and associated emotional/behavioral changes alters with age making any treatment tedious.

Case Description: Chair side full mouth rehabilitation of three-and-a-half-year-old male child who is a known case of Prader-Willi syndrome using behavioral modification techniques making it challenge to the pedodontist to treat the child.

Conclusion: Successful identification of the syndrome and early treatment of the associated dental problem and implementation of preventive strategies by application of appropriate behavioral management techniques will help the children to improve their quality of their life.

Clinical significance: Modest use of Behavior modification in handling a child who is a known case of Prader-Willi syndrome (PWS) and thus insisting that not all children who are known cases of PWS essentially be treated under General Anesthesia.
\end{abstract}

\section{Introduction}

Prader-Willi syndrome (PWS) is a rare neuro-developmental disease caused by abnormalities on chromosome 15q11.2-q13. Estimated occurrence is 1:15,000 live births. Clinically the condition is expressed as a dysmorphic syndrome that principally affects the central nervous system and has a particular predilection for the hypothalamus. Original description was made by Prader, Labhart, and Willi in 1956 [1].

The syndrome has a characteristic phenotype which includes neonatal and infantile hypotonia, early feeding problems (naso-gastric tube feeding for more than two months), childhood onset hyperphagia, obesity, short stature associated with growth hormone $(\mathrm{GH})$ deficiency, high pain threshold, learning disabilities and behavioral problems. Excessive daytime sleepiness and sleep apnea are common in individuals with PWS. A narrow forehead, almond-shaped eyes, downturned corners of the mouth and a thin upper lip are characteristic facial features. Varying degrees of oral motor dysfunction is common in individuals with PWS.

Thick viscous saliva has been reported to be a consistent finding in PWS and a diagnostic indicator of PWS in neonates. Decreased salivary flow rates and increased amounts of salivary ions and proteins have been detected, along with Enamel defects and Progressive dental tissue loss (tooth wear) [2]. Other reported oral findings include delayed teething; excessive number of teeth and microdontia high arched palate, bruxism and tooth position anomalies are all common. There is an increased risk of caries owing to eating problems and mouth dryness have been reported in literature [3].

\section{Case description}

The male patient aged three and half years diagnosed with PWS was presented with his parents to Department of Pedodontics and Preventive Dentistry at Ragas Dental College and Hospital with the chief complaint of pain in the right lower posterior teeth (Figure 1). He was referred by an endocrinologist from Sri Ramachandra Hospital where he has been receiving treatment for hypothyroidism for past two and half years and the reports showed he had no glucose intolerance.

${ }^{\star}$ Correspondence to: Vignesh Guptha Raju, Department of Pedodontics and Preventive Dentistry, Karpaga Vinayaga institute of dental sciences, Tamil Nadu, India, E-mail: vigneshguptha88@gmail.com

Key words: prader-willi syndrome, obesity, oral findings, behavioral management techniques

Received: March 21, 2019; Accepted: April 05, 2019; Published: April 08, 2019 


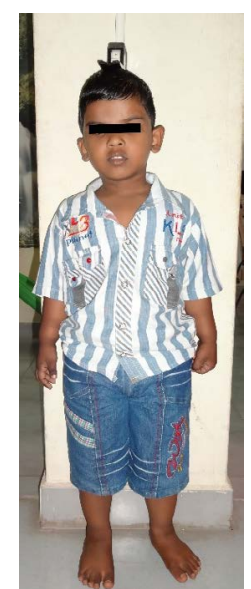

Figure 1. Picture representing a three-and-a-half-year-old male patient who is a known case of Prader-Willi syndrome

History (obtained from the patient and his mother) revealed that patient had pain of throbbing and intermittent type, which aggravated during mastication of food and patient gave history of hypothyroidism, allergy to amoxicillin. Patient dint have any other oral habits.

General examination of patient revealed, height as 96 centimeters, weight as 19 kilograms and BMI score of 20.4. When placing the BMI for age at greater than the $99^{\text {th }}$ percentile for a child of similar age and sex the child was obese. Extra-oral examination of patient revealed he had almond shaped eyes, down turned corners of mouth, thin hypotonic upper lip with lip incompetence, square facial form with anterior divergent and palpable right-left submandibular lymph node.

Intra oral examination of the patient revealed number of teeth as 20 with flush terminal plane molar relation, hypocalcified enamel in $53,52,63,62$, poor oral hygiene \& viscous saliva (Figure 2a-2c). OPG revealed root resorption in 85 which was confirmed with IOPA. IOPA of $74,84,75,85,54$, and 64 revealed radiolucency approximating the pulp.

Treatment was started with diet counselling and instructions owing to the surplus body weight corresponding to the age and prophylactic recommendation in order to maintain the child's dental health close to normal, followed by behavioral modification techniques like modelling, systematic desensitization, tell show do and distraction were employed to obtain child's cooperation during treatment.

After achieving child's trust during initial appointments, treatment sessions were started with composite restorations in anterior teeth (51, $52,53,61,62$, and 63) (Figure 3) and then posterior teeth $(55,65)$. During subsequent visits once, appropriate behavior was obtained pulp therapy followed by stainless steel crowns were given (Figure 4a) $(54,64,74,75 \& 85)$. Finally, 84 were extracted and crown \& loop space maintainer was luted in 85 (Figure 4 b). Oral prophylaxis was performed at the last treatment sessions followed by topical fluoride application and oral hygiene instructions were given. The child was initially reviewed once in three months for one year followed by review at six months interval and found to be with no new lesion and had good oral hygiene.

\section{Discussion}

The diagnostic criteria for Prader-Willi Syndrome are classified according to their significance. Major PWS criteria include neonatal hypotonia, feeding problems in infancy, characteristic facial features, excessive weight gain, hypogonadism, developmental delay and hyperphagia leading to obesity. Minor criteria include decreased fetal activity, behavioral and emotional problems (stubbornness, obsessive-compulsive behaviors, hyperactivity, and aggressiveness), self-destructive behaviors (e.g. picking at the skin), mental retardation, delayed speech and memory development, sleep disturbances, short stature, hypopigmentation of the skin and hair, small hands and diminished secretion of thick and sticky saliva. Supportive findings such as scoliosis, osteoporosis and a high pain threshold are also used in diagnosing PWS [4].

Thyroid axis dysfunction seems to be a frequent feature in infants with PWS. This is especially important since neonatal TSH screening is not an accurate tool for diagnosing thyroid axis dysfunction. Free T4, T3, and TSH levels should be monitored regularly in PWS children, particularly during GH treatment [5].

Most reports on the dental findings in PWS have focused on the presence of rampant caries, tooth wear, delayed tooth eruption, and hypoplastic enamel; here a case report of three and half year's boy diagnosed with PWS was presented. Full mouth rehabilitation was done for this child at chair side using behavior management techniques. Whereas some were treated under General Anesthesia because of their behavioral problem [6].

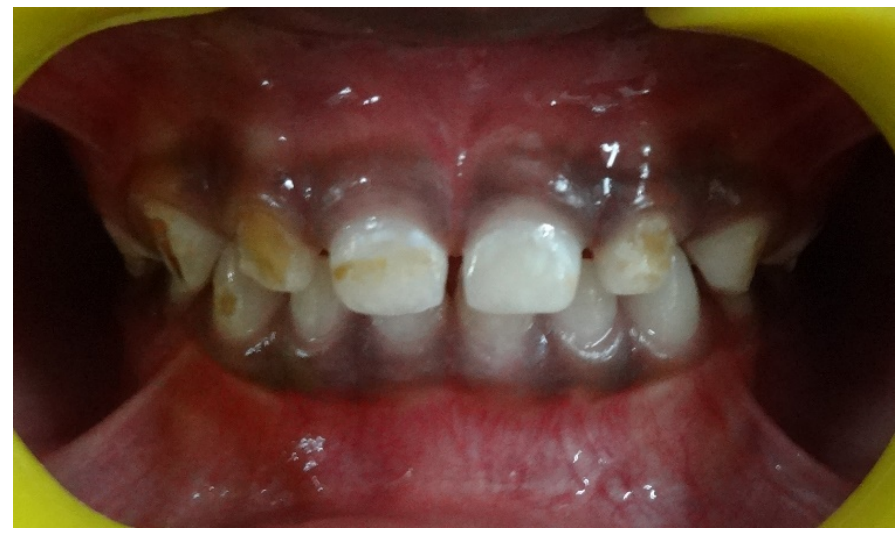

Figure 2a. Clinical picture representing the anterior teeth. Note enamel wear in 52,53,62,63

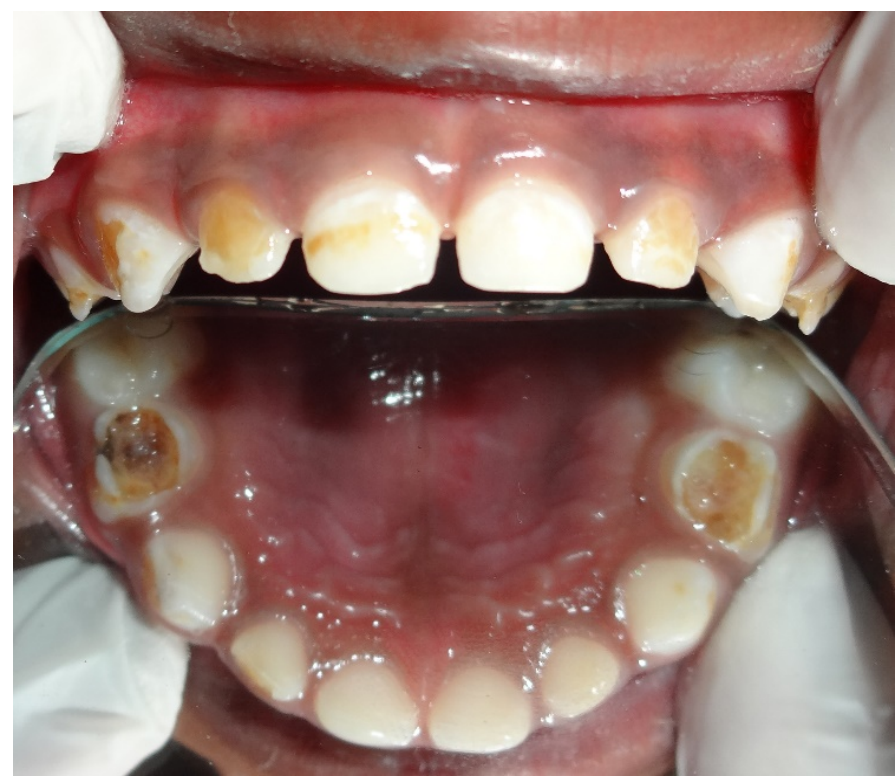

Figure 2b. Intra oral picture showing maxillary arch 


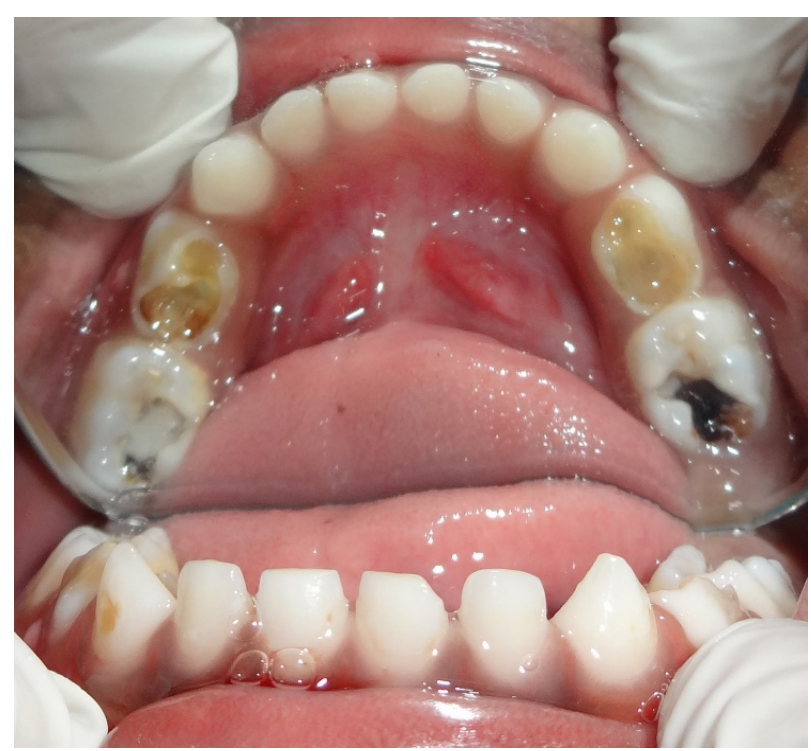

Figure 2c. Intra oral picture of Mandibular arch with dental caries in 74,75,84\&85.

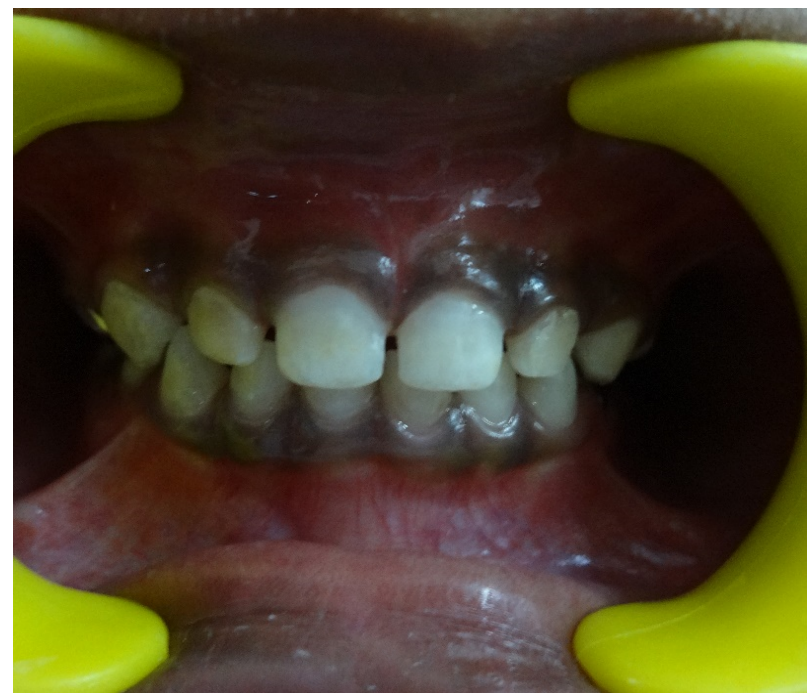

Figure 3. Picture showing anterior teeth after composite restoration

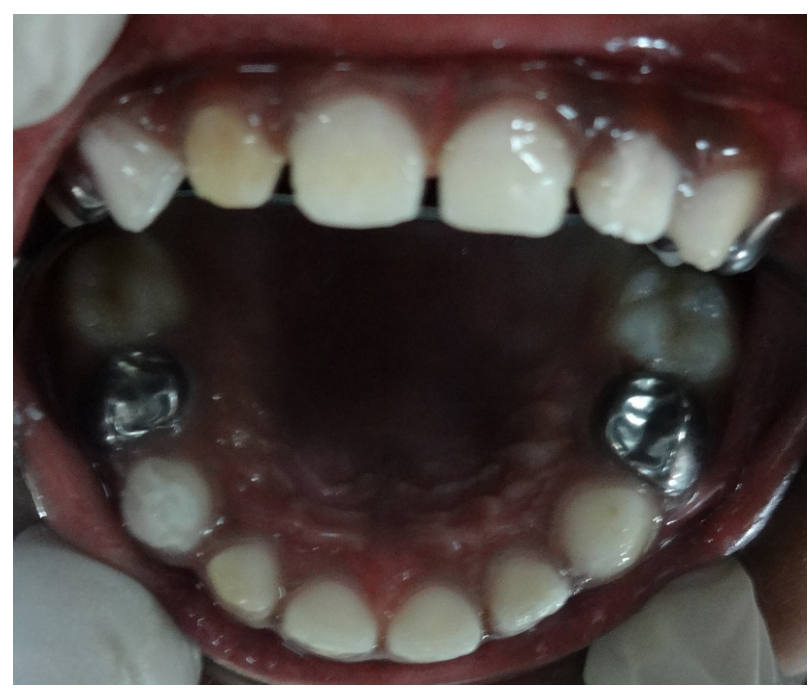

Figure 4a. Post-operative Maxillary occlusal view

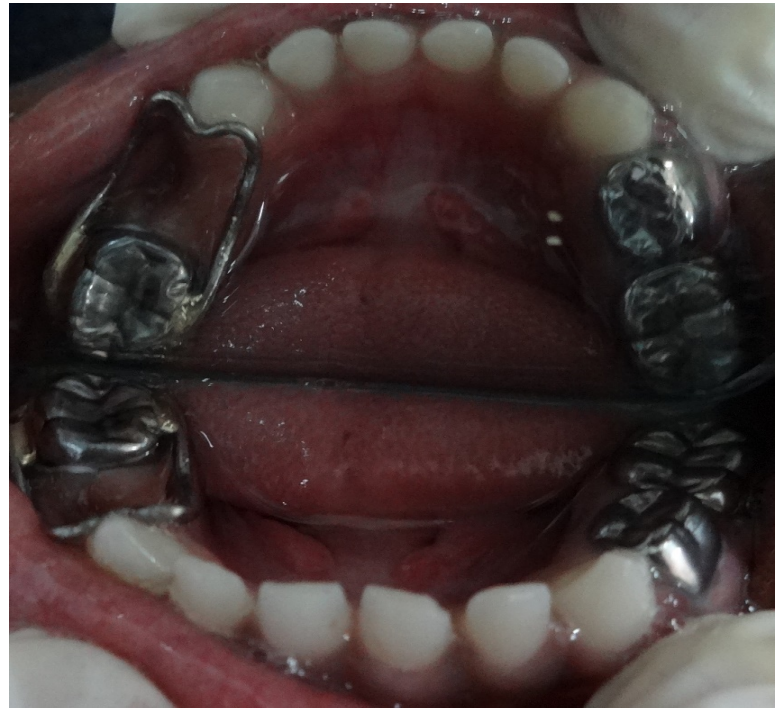

Figure 4b. Post-operative Mandibular occlusal view showing crown and loop space maintainer in relation to 84 and SS crown in $74 \& 75$ a

Prevention with regular professional cleaning as part of a comprehensive oral preventive program is particularly important for individuals with PWS. Monitoring of reduced salivation and increased caries by one year of age should be considered. Training in oral motor skills and extra stimulation may be relevant [4]. Eruption sequence pattern should be reviewed if necessary preventive strategies application of Pit and Fissure sealants for permanent molars as and when observed. Speech, language and communication training are often justified. Snoring problems should be followed up by a physician. Tooth grinding should be followed up and be managed with a soft splint when necessary. An orthodontist should be consulted between the ages of seven and nine in order to identify dental aberrations or malocclusions and to plan any necessary orthodontic treatment.

\section{Conclusion}

Similar to other genetic disorders, PWS shows significant changes in the patient's oral environment. It is with the deliberant diagnosis and legitimate treatment rendered to the child by the dentist that helps to maintain the child's oral cavity healthy. Also, proper behavior management is vital for treating a child with PWS.

\section{Clinical significance}

As a part of treating a patient with PWS, mere treatment of the conditions present doesn't suffice the completion of treatment. In order to maintain the oral health status of the patient stable, routine re-visits to the dentist will help in proper maintenance of the oral cavity as a whole. In other words, regular follow-up and treatment (if necessary) to the patient like monitoring the developing occlusion and early detection and management of caries is necessary and not mere management of the patient's emotional condition for a particular treatment.

\section{Conflicts of interest}

There is no conflict of interest for this manuscript submitted.

\section{References}

1. Donaldson MD, Chu CE, Cooke A, Wilson A, Greene SA, et al. (1994) The PraderWilli syndrome. Arch Dis Child 70: 58-63. [Crossref]

2. Ronnaug Saeves, IvarEspelid, Kari Storhaug, LeivSandvik, Hilde Nordgarden (2012) Severe tooth wear in Prader-Willi syndrome. A case-control study. BMC Oral Health 12: 12. [Crossref] 
Raju VG (2019) A three-and-a-half-year-old patient with Prader-Willi syndrome: a case report on dental management with behavioral modification

3. Umapathy T, Premkishore K, Mithesh D Kathariya, Sridhara KS, et al. (2013) Oral and General Findings: Management of Prader-Willi Syndrome. J Indian Acad Oral Med Radiol 25: 30-34.

4. Sung Yoon Cho, Dong-Kyu Jin (2012) Issues in Infants with Prader-Willi Syndrome: Special Review on Early Dietary Intervention and Early Use of Growth Hormone. Ann Pediatr Endocrinol Metab 17: 145-152.
5. Aneta Witt, Dorota Olczak-Kowalczyk, Maria Ginalska-Malinowska, Malgorzata Zadurska (2011) Oral Findings in Prader-Willi Syndrome-Case Report. Dent Med Probl 48: 103-107.

6. Ki Un Song, Ok Hyung Nam, Mi Sun Chul Choi, Hyo-Seol Lee (2015) An 18-yearold patient with Prader Willi syndrome: A case report on dental management under sedation and general anesthesia. J Dent Anesth Pain Med 15: 251-255. [Crossref]

Copyright: @2019 Raju VG. This is an open-access article distributed under the terms of the Creative Commons Attribution License, which permits unrestricted use, distribution, and reproduction in any medium, provided the original author and source are credited. 